\title{
Preuves fondées sur la pratique, pratiques fondées sur la preuve : distinction et mise en synergie
}

Gérard Sensevy, Jérôme Santini, Didier Cariou et Serge Quilio

\section{OpenEdition}

1 Journals

Édition électronique

URL : http://journals.openedition.org/educationdidactique/3400

DOI : 10.4000/educationdidactique.3400

ISSN : 2111-4838

Éditeur

Presses universitaires de Rennes

\section{Édition imprimée}

Date de publication : 21 décembre 2018

Pagination : 111-125

ISBN : 978-2-7535-7711-4

ISSN : 1956-3485

Référence électronique

Gérard Sensevy, Jérôme Santini, Didier Cariou et Serge Quilio, «Preuves fondées sur la pratique, pratiques fondées sur la preuve : distinction et mise en synergie », Éducation et didactique [En ligne], 12-2 | 2018, mis en ligne le 21 décembre 2018, consulté le 07 janvier 2021. URL : http:// journals.openedition.org/educationdidactique/3400; DOI : https://doi.org/ERREUR PDO dans / localdata/www-bin/Core/Core/Db/Db.class.php L.34 : SQLSTATE[HY000] [2006] MySQL server has gone away 


\title{
PREUVES FONDÉES SUR LA PRATIQUE, PRATIQUES FONDÉES SUR LA PREUVE DISTINCTION ET MISE EN SYNERGIE
}

\author{
Gérard Sensevy \\ ESPE de Bretagne - UBO, CREAD \\ Jérôme Santini \\ université Côte d'Azur - ESPE académie de Nice, URE LINE \\ Didier Cariou \\ ESPE de Bretagne - UBO, CREAD \\ Serge Quilio \\ université Côte d'Azur - ESPE académie de Nice, URE LINE
}




\section{INTRODUCTION}

Dans ce commentaire à l'article d'A. S. Bryk (2017), nous proposons une lecture de ce texte centrée sur l'expression «preuve fondée sur la pratique » (practice-based evidence) ${ }^{1}$. Nous précisons tout d'abord ce que peut signifier, selon nous, cette expression. Nous essayons ensuite de brièvement définir dans quelle épistémologie de la pratique, et en particulier de la pratique scientifique, une telle conception s'inscrit. Nous nous attachons ensuite aux conséquences de cette conception sur les relations entre la pratique et la recherche, à travers l'idée d'ingénierie coopérative. Nous terminons notre commentaire en insistant sur la nécessité de mise en synergie de ce que nous appelons des preuves anthropologiques et des preuves statistiques.

\section{QU'EST-CE QU'UNE PREUVE FONDÉE SUR LA PRATIQUE ?}

L'article de A. S. Bryk est pour nous essentiel, pour de multiples raisons. La première d'entre elles, de notre point de vue, consiste dans son usage de l'idée de preuve fondée sur la pratique (practicebased evidence). Nous expliciterons l'importance que nous lui attribuons dans la suite de ce commentaire, mais il faut déjà noter un fait central. Cette notion de preuve fondée sur la pratique, dont Bryk nous semble faire un usage si fructueux pour la recherche en éducation, a d'abord été proposée en médecine. Elle constitue une production de la méthode clinique. Les chercheurs qui l'ont élaborée l'ont fait à la fois en réaction et en complément à une recherche attachée à déterminer des « pratiques fondées sur les preuves » (evidence-based practice ${ }^{2}$ ).

Sans aucunement nier l'intérêt d'une telle orientation, ces chercheurs ont notamment tenté de retrouver, à travers la notion de preuve fondée sur la pratique (practice-based evidence), le sens pratique des médecins qui pouvaient prendre des décisions, de type clinique, qui ne relevaient pas directement de preuves statistiques (cf. notamment Barkham et Melior-Clark, 2003 ; Green, 2008³). Arrêtons-nous un instant ici. Les pratiques fondées sur des preuves (evidence-based practice) sont des pratiques fondées sur des preuves statistiques. L'article de A. S Bryk, et les travaux accomplis notamment en clinique médicale sur lesquels il s'appuie, et plus généralement les recherches philosophiques et épistémologiques nous incitent à penser qu'il existe d'autres preuves que statistiques ${ }^{4}$. En particulier, il existe des preuves fondées sur la pratique, c'est-à-dire, nous le verrons, sur la connaissance pratique de la pratique ${ }^{5}$, que nous nommerons dans ce qui suit « preuves anthropologiques ${ }^{6} »$. En quoi ce type de preuve consiste-t-il ?

Selon nous, on peut établir deux catégories de preuves fondées sur la pratique.

La première catégorie renvoie à ces preuves, ces «évidences", dont on a une conviction profonde, intuitive, immédiate, née de l'habitude, d'une forme de connaissance directe de la vie, d'une forme de vie. Dans son Précis de psychologie, William James (1890, traduction française 1909, p. 565-567) utilise un exemple frappant. Il analyse un sentiment et un comportement que nous connaissons tous. C'est l'hiver, nous avons dormi dans une pièce froide, « dans une chambre sans feu », nous dit William James, et nous devons nous lever. La chaleur du lit nous incite à rester au chaud. D'une certaine manière, nous avons l'impression, physique, que nous ne nous pourrons jamais nous lever, quelles que soient les tâches qui nous attendent. «Comment se fait-il que l'on se lève enfin ? », demande James. «Le plus souvent, on se lève sans l'ombre d'une lutte intérieure ou d'une décision expresse : on s'aperçoit tout soudain qu'on s'est levé ». C'est qu'une idée nous a traversé, qui a échappé et fait échapper à la «lutte d'une vive conscience du froid et d'une vive conscience de chaleur qui tout à l'heure paralysait notre activité, et réduisait l'idée de se lever à la simple condition d'un désir, au lieu de la laisser s'actualiser en une volition efficace ».

Cet exemple est emblématique d'une première catégorie de preuve fondée sur la pratique, une première catégorie de preuve anthropologique. On saisit pourquoi nous utilisons ce dernier terme. Il s'agit, nous l'avons dit, de preuves intuitives, immédiates, nées de la connaissance de la vie, qu'on postule propres (ou bien susceptible de le devenir) à tout être humain. Elles sont donc anthropologiques. Comme la connaissance de la vie pratique sur laquelle elles reposent est le propre de tout être humain, ces preuves constituent une évidence partagée. D'une certaine façon, elles mettent tout le monde d'accord. Comme l'exemple de James, elles renvoient à une expérience commune. Il n'est d'ailleurs pas indifférent de noter que William James luimême fait ici de cette expérience commune un usage 
de preuve ${ }^{7}$. C'est bien cette connaissance anthropologique de la pratique humaine (ici, celle du lever), qu'il utilise pour garantir $^{8}$ une théorie de la volition. Il est encore plus intéressant de noter que la citation que nous faisons des propos de James est elle-même une citation. Nous l'avons trouvée dans le livre de G. Rizzolatti et C. Sinigaglia, Les neurones miroirs (2008, p. 153) qui, eux-mêmes, citent longuement l'argument de James, en particulier pour souligner le fait que « la plupart de nos gestes quotidiens ne nécessitent pas une décision particulière, un fiat licite $»$. Une telle conception prend place dans la théorie de l'imitation qu'ils développent dans leur livre, théorie qu'ils garantissent à la fois par des preuves d'imagerie cérébrale, et des preuves anthropologiques comme celles de l'argument jamesien. Notons dès à présent qu'une telle preuve anthropologique est à la fois fondée sur des particuliers (le sentiment de froid, la « lutte » entre le chaud et le froid, qui d'abord paralyse et qu'on oublie ensuite) et sur une temporalité, une durée spécifique (celle du lever). Ces particuliers, dans leurs durées, renvoient à des institutions (ici, l'institution du lever) et particulièrement, pour reprendre l'expression et la conceptualisation de Vincent Descombes, à des institutions du sens (Descombes, 1996, 20149).

Bien entendu, ce type de preuves, comme tout autre, a des défauts. Fondé sur la connaissance de la pratique dont peuvent témoigner les humains, il peut par exemple faire prendre des allant-de-soi pour des preuves. De fait, cette première catégorie de preuve anthropologique a la force et le défaut des proverbes (il ne serait d'ailleurs pas très difficile de convertir l'apologue de James en proverbe, par exemple: "Même quand la chambre est glacée, on finit toujours par se lever $»$ ). Les proverbes constituent une forme de sagesse de la pratique, mais ils peuvent aussi empêcher de penser. Il faut donc être conscient et prévenu de ces défauts liés aux preuves anthropologiques (fondées sur la connaissance pratique de la pratique), mais cet avertissement vaut pour tout type de preuve, pour les preuves statistiques comme pour toutes les autres ${ }^{10}$.

Nous avons postulé deux catégories de preuves fondées sur la connaissance de la pratique. Le premier type de preuve (du type de l'exemple de James) renvoie à une connaissance universelle, dont tout être humain dispose ou peut disposer rapidement s'il traverse l'expérience adéquate. Le second type de preuve renvoie lui à une connaissance du même genre, mais qui est fondée sur une habileté, une capacité, un art, un skill ${ }^{11}$. Il s'agit d'une preuve qui fait sens pour le connaisseur pratique. Ce type de preuve joue un rôle majeur au sein de nombreuses activités humaines, en particulier dans l'expérience esthétique. Considérons l'exemple suivant, que l'on doit à Peter Brook (1968, p. 76), explicitant l'un des exercices qu'il demandait de travailler à des comédiens :

Un des moments les plus intéressants, ce fut durant un exercice où chaque membre du groupe devait représenter un enfant. Naturellement, les acteurs, les uns après les autres, imitèrent un enfant : ils se baissaient, se tortillaient, ou poussaient des cris aigus. Le résultat était lamentable. Puis l'acteur le plus grand du groupe s'avança, et sans le moindre mouvement physique, sans essayer d'imiter le langage enfantin, présenta de façon totale, à la satisfaction de tous, l'idée qu'on lui avait demandé de faire passer. Comment ? Je ne peux le décrire ${ }^{12}$; cela se passa dans une sorte de communication directe et pour ceux qui étaient présents seulement. C'est cela que certains théâtres appellent la magie, d'autres la science, mais c'est la même chose. Une idée invisible était représentée de façon adéquate.

L'exemple qui précède est pour nous important. Nous saisissons ici certaines caractéristiques de cette "deuxième catégorie » de "preuve anthropologique $»$. Comme la première catégorie, elle est « immédiate ${ }^{13}$ » (sans « médiation »), et produit une conviction commune. Les acteurs s'accordent, et ils s'accordent immédiatement pour trouver adéquate la représentation de l'enfant par l'un d'entre eux. Mais contrairement à la première catégorie, cette forme de preuve ne relève pas seulement d'une connaissance, d'une capacité, partagées par tous. Cette preuve renvoie à une habileté, à une capacité, un skill, un art de faire (de Certeau, 2010) qui témoigne d'une forme d'accomplissement. Nous faisons l'hypothèse que tout connaisseur pratique (sans limiter nullement cette notion aux arts), en produisant cette forme d'accomplissement, produit une preuve, une évidence. Cette preuve peut se concevoir comme une sorte de théorème d'existence. L'acteur qui fait l'exercice de P. Brook montre qu'il est possible de " représenter l'enfant», en sachant que celle ou celui qui veut "représenter l'enfant » d'une manière analogue devra s'inscrire dans un espace-temps spécifique et refaire, en la renouvelant pour lui, 
l'expérience de cette représentation. L'acteur qui fait l'exercice actualise concrètement la virtualité de cette représentation. Ce type de garantie jouera un rôle majeur, dans la transmission par exemple. Ajoutons donc ici l'élément suivant : ce qui fait preuve, ce qui fait garantie, pour le connaisseur, joue un rôle fondamental dans la pratique. Il existe ainsi une dynamique de garantie dans la pratique humaine. On se fonde sur ce qui est garanti en allant plus loin. C'est en comprenant mieux, peu à peu, dans le sensible et dans le rationnel, à quoi tient la « représentation de l'enfant » dont l'acteur a été capable, qu'on maîtrisera davantage l'art de la représentation.

Bien entendu, comme tout type de preuve, ce type de preuve anthropologique (les preuves liées aux capacités de connaisseurs pratiques) est faillible. Le connaisseur pratique, aussi habile soit-il, peut se tromper, et l'histoire des pratiques, par exemple celle des pratiques scientifiques, fourmille d'exemples de telles erreurs ${ }^{14}$. Cela signifie donc que toute analyse du processus de preuve, de garantie, doit intégrer une analyse de faillibilité propre au type de preuve dont on fait usage.

Dans ce qui précède, nous avons tenté de donner un statut à cette notion de preuve fondée sur la pratique, ou plus exactement sur la connaissance pratique de la pratique. Nous voulons maintenant contextualiser cette notion à l'éducation. Nous soutenons que nous pouvons trouver, au sein du processus d'éducation, des preuves du même type.

Nous trouvons d'abord des preuves de type « connaissance commune du processus d'éducation », analogues à celles dont nous avons donné l'emblème avec le lever matinal évoqué par James.

Prenons par exemple le cas de l'effet Topaze. Guy Brousseau (1998), dans son étude de la relation didactique, s'est appuyé sur des œuvres littéraires (Topaze, de Marcel Pagnol, mais aussi Le Bourgeois gentilhomme, de Molière, pour l'effet Jourdain) afin de déterminer certains dysfonctionnements de cette relation. L'effet Topaze renvoie à un processus bien connu de tout professeur. Lorsqu'un élève a du mal à répondre à une question du professeur, celui-ci peut l'aider à trouver la réponse. Il peut, comme on dit, le « mettre sur la voie » de la réponse. Mais cet effort peut déraper. Le professeur peut en dire (beaucoup) trop. C'est ce qui arrive, dans la pièce de Pagnol, à Topaze, l'instituteur. Celui-ci dicte la phrase « Des moutons étaient en sûreté dans un parc ». Un élève écrit « les mouton», sans le «s » final qui marque le pluriel. Voici un court extrait de la pièce :

TOPAZE, il dicte en se promenant « Des moutons... Des moutons... étaient en sûreté... dans un parc ; dans un parc. (Il se penche sur l'épaule de l'Élève et reprend.) Des moutons... moutonss... (L'Élève le regarde ahuri.) Voyons, mon enfant, faites un effort. Je dis moutonsse. Étaient (il reprend avec finesse) étai-eunnt. C'est-à-dire qu'il n'y avait pas qu'un moutonne. Il y avait plusieurs moutonsse. »

Lélève le regarde, perdu.

On voit comment Topaze fait bien plus qu'aider à trouver la réponse. Comme on dit, il la « souffle» à l'élève. Il est d'ailleurs intéressant de constater qu'après avoir donné la réponse en soulignant oralement la terminaison des mots « moutons » et " étaient ", Topaze essaie tout de même de faire son métier de professeur, et d'expliquer la règle du pluriel, tout en maintenant "l'effet Topaze * ( « C'est-à-dire qu'il n'y avait pas qu'un moutonne. Il y avait plusieurs moutonsse »). La réaction de l'élève, d'abord « ahuri », puis « perdu », est édifiante.

Qu'est-ce que l'effet Topaze, donc ? C'est ce qui se passe lorsque le professeur donne à l'élève les moyens de répondre à la question qu'il pose, sans que cet élève ne s'appuie sur la connaissance adéquate (ici le pluriel des noms et celui des verbes) pour répondre à cette question.

On conçoit ici qu'on a affaire à une connaissance pratique de la pratique. On pourrait dire, sans jeu de mot, une connaissance grammaticale de la pratique. Dans la grammaire de l'action professorale, on peut relever cette structure : le professeur est confronté au risque de l'effet Topaze, qui est d'une certaine manière en même temps inévitable. On pourra d'ailleurs faire l'hypothèse que l'explicitation de cet effet, son étude, et sa caractérisation aideront à mieux comprendre l'art d'enseigner. Quelle est la preuve de l'effet Topaze? Il s'agit d'une preuve anthropologique. Tout connaisseur pratique de la pratique d'enseignement appréhende cette preuve, parce qu'elle renvoie à l'expérience de tout professeur (et de tout élève aussi bien).

Nous pouvons ainsi désigner l'effet Topaze comme un exemple de caractérisation de la relation didactique qui repose sur une preuve anthropolo- 
gique du premier type, au sens où nous l'avons défini ci-dessus.

Quel peut être le second type de preuve anthropologique dans les recherches en éducation ? Celui-ci, nous l'avons précisé, ne relève pas seulement d'une connaissance partagée par tous (comme c'est le cas de l'effet Topaze, une fois qu'il a été explicité et exemplifié). Ce second type renvoie à un art de faire (de Certeau, 2010), qui témoigne d'une forme d'accomplissement. Il s'agit, on l'a vu, de la preuve d'un connaisseur pratique. En éducation, elles sont constantes, même si elles ne sont pas souvent reconnues. Par exemple, considérons le domaine des preuves d'efficacité d'un enseignement. On peut avoir bien entendu des preuves statistiques d'une telle efficacité - elles sont importantes, et nous reviendrons sur ce point. Mais il existe aussi des preuves anthropologiques, des preuves de connaisseurs pratiques de cette efficacité. Tout professeur expérimenté, et tout chercheur suffisamment familier de la pratique d'enseignement, peut reconnaître dans une production effective d'élève (orale ou écrite) la manifestation claire d'une connaissance, par exemple d'une connaissance épistémiquement dense. Mieux, il peut identifier un dialogue, au sein d'un collectif d'élèves, qui témoigne que ce collectif s'exprime sur la base d'un arrière-plan épistémique " de haut niveau ». Il peut ainsi appréhender, dans une classe donnée, un collectif de pensée qui témoigne d'une activité intellectuelle collective épistémiquement dense. Ce professeur (ou ce chercheur) peut d'ailleurs, dans un collectif de professeurs et/ou de chercheurs, partager cette reconnaissance, cette identification, l'expliciter, la discuter, chercher à améliorer la densité épistémique reconnue, la développer, etc. Ces « preuves d'efficacité », du type anthropologique donc, seront des preuves de connaisseur pratique, des preuves culturelles, au sens où elles viendront à participer de la culture épistémique (Knorr-Cetina, 1999) d'un collectif. De la même façon qu'un comédien reconnaît dans une performance théâtrale une qualité particulière, le professeur ou le chercheur pourront reconnaître dans le travail des élèves, dans leurs productions concrètes, dans le dialogue qu'ils entretiennent, des qualités particulières. C'est leur expérience, dans la durée, de l'enseignement, et la connaissance de l'enseignement que cette expérience - et son étude leur en aura permise, qui leur permettront de distinguer dans la pratique des élèves ce qui témoigne de leur avancée, individuelle et collective, c'est cette expérience et cette connaissance - cette culture qui leur permettront d'identifier ces qualités pour les faire fructifier.

Avant de poursuivre, notons ici que les preuves anthropologiques dont nous tentons de définir les contours sont organiques à certaines disciplines. Carlo Ginzburg, notamment dans un article fameux (Ginzburg, 1987), avance ainsi l'idée de deux paradigmes de recherche. Un paradigme galiléen, dans lequel les formalismes mathématiques jouent un rôle majeur, et un paradigme indiciaire, qui repose sur la détermination d'indices. Des héros de ce paradigme indiciaire, nous dit Ginzburg, sont notamment Giovanni Morelli, un historien d'art ${ }^{15}$, Sherlock Holmes, et Sigmund Freud, mais aussi les trois princes de Serendip ${ }^{16}$. Ce paradigme prend ses racines, selon Ginzburg, dans l'activité du chasseurcueilleur de la Préhistoire, qui se penche sur les traces laissées par les animaux qu'ils chassent. Ainsi, une branche cassée dans un sous-bois pourra faire signe. Il est très important de saisir que la perception de ce signe ne renvoie pas à la performance ou la compétence d'un seul individu - même si tous les individus ne sont pas également habiles. Il renvoie à un collectif de pensée, qui a forgé un style de pensée (Fleck, 2005), une manière de concevoir et de percevoir dans le collectif - ici, le collectif de chasseurs. L'expérience transforme l'individu et il devient capable de voir de nouvelles formes ; les connaisseurs pratiques de la pratique se reconnaissent entre eux car ils partagent un nombre d'expériences suffisamment analogues pour se comprendre.

Ce paradigme indiciaire pourrait être avantageusement rapproché du paradigme clinique, tel que Foucault (1963) décrit son avènement en médecine (Sensevy, 1999). Le paradigme clinique, comme le paradigme indiciaire, suppose une observation attentive des choses-mêmes, et la détermination de constellations d'indices qui fourniront une étiologie. Les preuves indiciaires, comme les preuves cliniques, sont des preuves anthropologiques du deuxième type, des preuves anthropologiques (culturelles donc) de connaisseurs pratiques. Elles reposent sur l'art de connaisseurs pratiques (par exemple le détective, le chasseur-cueilleur, le médecin, l'historien d'art, etc.) et sont d'autant plus puissantes et convaincantes que l'art du connaisseur pratique est grand, et que le style de pensée dans lequel il s'inscrit est efficace.

Il est à noter par ailleurs, ainsi que le montre Foucault, que le paradigme clinique se développe 
en médecine en même temps que l'anatomopathologie - qui repose sur d'autres types de preuves - en s'appuyant étroitement sur elle. Cela peut nous inciter à concevoir des recherches au sein desquelles la mise en synergie de différents types de preuves constitue un objectif essentiel. Nous reviendrons sur ce point à la fin de ce commentaire. Mais nous soutenons que cette mise en synergie nécessite un travail épistémologique, qui consiste notamment à repenser la notion de contexte, et dont nous présentons maintenant quelques linéaments.

\section{PREUVES ET CONTEXTES}

Les preuves prennent leur sens dans un contexte. On pourrait croire qu'il s'agit ici d'une spécificité des sciences de la culture, alors que les sciences de la nature seraient indépendantes des contextes. On peut lire le travail de l'épistémologue et philosophe des sciences, Nancy Cartwright, pour se convaincre du contraire. Celle-ci (notamment 1983, 1999) montre que d'une certaine manière " comment les lois de la nature mentent ${ }^{17}$ ", au sens, notamment, où elles sont vraies seulement sous certaines conditions extrêmement abstraites, et rigoureusement fausses dans toute situation concrète. Cartwright identifie ainsi une relation particulière entre « lois » et modèles : les modèles, en concrétisant les lois, permettent de leur donner une efficacité, toujours limitée à un contexte particulier, que Cartwright nomme « machine nomologique » (Cartwright, 1999). Il existe ainsi, nous dit la philosophe des sciences, une relation entre loi et modèle qui est du même type que celle entre la morale d'une fable et le récit qui lui correspond. "La raison du plus fort est toujours la meilleure » est un énoncé général, mais tellement abstrait qu'on aurait bien du mal à le comprendre s'il n'était réduit à un particulier, une histoire, par exemple celle du Loup et l'agneau (Lefeuvre, 2018). De même, « F = ma » décrit la première loi de Newton, mais cette « loi » n'est pas directement utilisable pour déduire le mouvement d'un pendule, par exemple. Pour faire cela, il faut réduire la loi au particulier d'une situation spécifique, celle du pendule, dont le modèle du mouvement sera une équation différentielle spécifique (pour une étude, dans le cadre didactique, de ces questions, cf. Sensevy et Santini, 2006 ; Sensevy, Tiberghien, Santini, Laubé et Griggs, 2008).
D'une certaine manière, donc, Cartwright nous aide à développer une conception "situationnelle » de la science, dans laquelle une opération fondamentale consiste dans cette réduction au particulier que les modèles peuvent opérer par rapport à ces énoncés plus généraux que sont les « lois». On peut alors penser une conception moniste des sciences, qui unit sciences de la nature et sciences de la culture, et qui supprime le dualisme entre sciences « nomologiques » et sciences « contextuelles » : les sciences de la nature, comme les sciences de la culture, sont contextuelles.

Quelles sont les conséquences de cette épistémologie sur une théorie de la preuve? Les preuves sont situationnelles. Elles ne prennent leur sens qu'à travers un modèle, qui, lui-même, tire son efficacité de son adéquation aussi précise que possible à une réalité décrite aussi précisément que possible. De ce point de vue, les preuves anthropologiques sont des modèles. Considérons l'effet Topaze, vu ci-dessus. On pourrait le considérer comme la réduction au concret d'une sorte de " loi » plus générale : dans une relation didactique, la nature de la transmission est dépendante de la communication qui s'établit entre professeur et élève. Cette loi - simple, de «bon sens », quoique non triviale - est très générale, beaucoup trop - en particulier pour qui veut agir en situation didactique. Le « modèle » de l'effet Topaze permet de la réduire au particulier d'une forme particulière de la relation didactique, qui survient lorsque le professeur fournit des signes à l'élève. La manière dont les signes fournis à l'élève sont en relation avec le savoir est essentielle pour comprendre la relation didactique et pour envisager son efficacité. Pour avoir du sens, donc, les preuves seront pensées sur l'arrière-plan du modèle, non de la « loi » seule. Par exemple, on montrera que tel comportement d'élève, qui aura utilisé improprement tel indice fourni improprement par le professeur, s'explique par l'effet Topaze. Celui-ci sera alors la conséquence de l'exercice d'une capacité - le fait que le professeur puisse orienter, pour le meilleur ou pour le pire, l'action de l'élève. D'une certaine manière, dans l'épistémologie moniste proposée par Cartwright, les capacités remplacent les lois (Cartwright, 1989, 1994).

Dans la perspective ici suivie, il est intéressant de constater que Nancy Cartwright a dédié beaucoup de ses travaux récents à la question de l'evidence-based policy et aux randomised controlled trials (Cartwright, 2009, 2010, 2011, 2013 ; Munro, 
Cartwright, Hardi et Montushi, 2017). Nous évoquerons rapidement, dans ce qui suit, l'un d'entre eux.

\section{PARAdigme DU TRAiteMENT, PARAdigme DE L'ACCOMPLISSEMENT}

Pour avancer dans l'exploration des deux grands régimes de preuves (statistiques et anthropologiques) que nous identifions ici, il nous paraît utile de poser une distinction entre deux paradigmes de recherche, celui du traitement, et celui de l'accomplissement ${ }^{18}$.

Dans l'expression " paradigme du traitement », nous entendons ce terme - traitement - au sens médical du terme. Nous pensons en particulier au traitement médicamenteux. Il faut souligner que l'evidence-based research, qui peut aider à concevoir de l'evidence-based practice, est issue de ce paradigme du traitement, fondé en particulier sur l'essai clinique randomisé (randomised controlled trials), qui aboutit la plupart du temps à la mesure d'efficacité d'un traitement. Mais qu'est-ce qu'un traitement médicamenteux ? Il est fondé sur ce qu'on pourrait appeler l'intégration passive d'une substance (ou de plusieurs). Par intégration passive, nous voulons signifier qu'il n'est pas possible, au moins consciemment, de réussir ou de rater la prise d'un traitement. On peut imaginer qu'on oublie de prendre un médicament, mais respecter la posologie du traitement revient à intégrer le traitement, sans qu'aucune habileté, capacité, skill, soit nécessaire pour cela. Il n'existe pas de réel savoir-faire nécessaire à la prise d'un traitement. Que le traitement soit efficace ou pas ne dépend pas de la façon dont on l'a intégré.

Dans le paradigme de l'accomplissement, les choses sont bien différentes. Par exemple, si l'on imagine une manière d'enseigner la lecture plus efficace que d'autres, et qu'on la propose à des professeurs, il faudra encore que les professeurs qui pratiquent cette manière d'enseigner réussissent sa mise en œuvre, dans un lieu et une durée propre à leur pratique. Ici, on peut concevoir deux manières de penser cette réussite. Il y a la réussite improbable de celui qui sait mal faire, et la réussite toujours contingente de celui qui sait bien faire - et qui donc a appris à bien faire - mais qui peut ne pas réussir, la situation, presque toujours complexe, ne le permettant pas ce coup-ci.

On conçoit aisément la différence cruciale entre les deux paradigmes.
Dans le paradigme du traitement, les sujets intègrent le traitement, sans effort autre que le suivi de la posologie, et l'on peut assez facilement juger des effets de ce traitement en utilisant la méthode expérimentale classique, puisque l'intégration du traitement est toujours satisfaisante.

Dans le paradigme de l'accomplissement, la réussite de l'accomplissement est toujours incertaine, et contingente. Puisqu'il y a savoir-faire, l'évaluation de l'effet de la manière d'enseigner sera soumise à la qualité du savoir-faire, à la nature, à la puissance et à l'exercice de la capacité qui organise la mise en œuvre. Elle sera soumise à l'habilité, aux skills - pour reprendre le terme anglais si pertinent, qu'on pourrait concevoir comme une capacité pleine de discernement - qui caractériseront l'accomplissement intelligent, et les intentions et stratégies d'enseignement que cette intelligence demande.

C'est à notre sens l'une des raisons majeures des résultats souvent fort décevants de la généralisation de « méthodes qui marchent » en éducation (notamment). Ces «méthodes qui marchent» marchent, peut-on penser, parce qu'elles sont mises en œuvre par des professeurs qui savent faire. La plupart du temps, s'ils savent faire, c'est qu'ils ont appris à faire, soit « sur le tas », soit dans une formation instituée. On voit bien donc que le paradigme de l'accomplissement doit intégrer non seulement le comportement que l'on pense efficace (enseigner telle chose de telles et telles façons), mais encore la manière dont les professeurs pourront accomplir adéquatement ce comportement. Ne pas faire cela revient à confondre les deux paradigmes, et à considérer l'accomplissement comme un traitement. Pour le dire autrement, la confusion entre les deux paradigmes peut se paraphraser ainsi : l'intégration par les professeurs (et les élèves conjointement !) de la « méthode d'enseignement » est considérée comme identique à la prise d'un traitement. L'intégration, en situation complexe, d'un système complexes d'intentions et de stratégies se retrouve alors malheureusement ramenée au suivi d'une posologie. On peut citer ici le travail de Cartwright et ses collègues, dans le domaine de l'aide aux familles pour la protection et le bien-être de l'enfance (child protection and welfare) :

We are arguing for the essential role of individual human skill in engaging with the complexity of the world and the tasks of understanding and helping families. This includes a radically different picture of 
where research fits into child welfare work from the currently dominant picture. The complexity of the social world means that there are severe limitations to how much we can develop general conclusions about what works in helping parents provide better and safer care for children and young people. Research should be seen as a valuable resource but it needs to be read critically, paying attention to negative as well as positive findings and reflecting on the extent to which its context resembles your own. Even when it looks a good bet that it might be helpful in your context, implementation needs to be monitored to see how it is interacting with the numerous other causal processes already operating or that may come up later in the process, perhaps producing unexpected results. At the same time, more attention needs to be given to the key role of deliberation ${ }^{19}$ in deciding what to do to help families. By its nature, deliberation cannot be turned into a formalized, deterministic process where there is one clearly "right" conclusion, but it can be done to varying standards. A better appreciation of how we reason provides guidance on how organisations can enhance the deliberative skills of their workforce. One factor is giving time for the task, i.e. recognizing its importance. Another is that openness about one's thinking allows others to challenge and support the process, reducing the risk of individual bias or limited experience.

(Munro, Cartwright, Hardi et Montushi, 2017, p. 145-146)

Pour percevoir et comprendre l'accomplissement que nous postulons ici, les preuves anthropologiques - en tant que modèles ${ }^{20}$ de comportement - jouent un rôle majeur. Elles seront fondées sur la description précise des comportements (intentions et stratégies d'enseignement) qui soustendent la "méthode » d'enseignement que l'on pense efficace. Cette conception a des conséquences épistémologiques importantes. En particulier, elle met au cour du processus d'élaboration scientifique et du processus de preuve des exemples emblématiques, que l'on peut considérer comme des exemples exemplaires (Kuhn, 1990) en devenir. Ces exemples emblématiques concrétisent à la fois les problèmes qui sont travaillés dans la recherche (par exemple dans une séquence d'enseignement), et la manière dont la recherche les pense et les traite.
Il faut donc que la recherche en éducation, selon nous, imagine des dispositifs fondés sur le paradigme de l'accomplissement. Nous allons consacrer les lignes qui suivent à décrire rapidement des prototypes de tels dispositifs.

\section{INGÉNIERIES COOPÉRATIVES : L'EXEMPLE D'ACE}

L'expression ingénierie coopérative ${ }^{21}$ désigne une manière de travailler qui réunit des professeurs et des chercheurs dans un travail produit au sein d'un même collectif. Le fonctionnement général est le suivant. Professeurs et chercheurs coopèrent dans l'élaboration d'une séquence d'enseignement. Ils la mettent en œuvre. La séance est ensuite évaluée, à travers cette mise en œuvre. Il s'agit alors d'en produire une nouvelle version, organisée autour de nouvelles hypothèses d'amélioration de la pratique. Cette nouvelle version est mise en œuvre à son tour. Le processus se développe par itérations successives, dans la longue durée - souvent sur plusieurs années $^{22}$. Il s'inscrit ainsi dans le paradigme de l'amélioration défendu par Bryk (2017).

Une ingénierie coopérative s'ancre dans quelques principes, dont nous décrivons ici certains.

Un principe de définition commune des fins de l'action. Celles-ci (par exemple les objectifs, les intentions, et les stratégies d'enseignement qui expriment cette action) sont déterminées le plus possible conjointement par les professeurs et les chercheurs. Cette définition commune des fins repose sur le travail collectif, intense et continu, du savoir qu'il s'agit de faire approprier aux élèves. Chercheurs et professeurs constituent ainsi un réseau (Nielsen, 2011), on pourrait dire un réseau épistémique ${ }^{23}$ qui évolue avec la mise en œuvre des versions successivement mises en œuvre.

Un principe de recherche de symétrie, qui récuse toute division du travail a priori entre chercheurs et professeurs ${ }^{24}$. Par exemple, après un long travail commun, la production d'une preuve anthropologique peut être initiée par un professeur comme par un chercheur. Par exemple, une "idée concrète » d'amélioration de la pratique peut être apportée par un chercheur. Une « idée abstraite » d'amélioration de la pratique par un professeur ${ }^{25}$.

Un principe d'assomption des différences entre les participants à l'ingénierie. La coopération profes- 
seurs-chercheurs n'implique pas un idéalisme ignorant des différences pratiques entre professeurs et chercheurs, dans le concret quotidien de leur action. $\mathrm{Au}$ contraire, chaque participant à l'ingénierie doit assumer son point de vue et le faire valoir, non seulement pour des raisons éthiques ou politiques, mais avant tout pour des raisons épistémologiques : c'est ainsi que les différences, la variété et les variations qu'elles peuvent produire, pourront bénéficier à l'entreprise collective.

Un principe de la posture d'ingénieur. Dans la production et l'évaluation des séquences, à certains moments, professeurs et chercheurs occupent les uns comme les autres la même posture d'ingénieur. Ici encore, le dualisme de la " théorie » et de la «pratique » se dilue. Il se dilue précisément dans cette posture d'ingénieur, qui demande à la fois au professeur et au chercheur de prendre une distance réelle avec sa pratique habituelle : le professeur doit davantage penser sa pratique sur l'arrière-plan de sa signification conceptuelle; le chercheur doit davantage penser sa conceptualisation sur l'arrière-plan de sa concrétisation pratique.

Pour donner un peu de chair à cette description bien abstraite, nous évoquerons rapidement un exemple concret d'ingénierie : l'ingénierie ACE.

Nous faisons vivre une ingénierie coopérative au sein du projet Arithmétique et compréhension à l'école élémentaire $\left(\mathrm{ACE}^{26}\right)$. Cette recherche présente les caractéristiques suivantes :

- professeurs et chercheurs co-construisent dans un même collectif une progression pour le $\mathrm{CP}$ et le CEl en mathématiques. Celle-ci prend la forme d'un texte d'enseignement qui décrit avec précision les séquences à mettre en œuvre. Ce texte d'enseignement est continûment renouvelé au fur et à mesure des mises en œuvre successives. Il intègre peu à peu la description d'exemples emblématiques (cf. ci-dessous) de moments cruciaux de la pratique ;

- cette progression a été évaluée, dans un processus d'evidence-based practice, quatre années consécutives (de 2012-2013 à 2016-2017), au CP et au CE1, sur la base d'un pré-test en début d'année, post-test en fin d'année, comparant un groupe « expérimental» (les professeurs mettant en ceuvre la progression ACE), et un groupe contrôle (des professeurs travaillant de manière habituelle). Chaque groupe rassemblait plus d'un millier d'élèves. Pour chacune des quatre évaluations, les «élèves ACE » ont réussi significativement mieux que les élèves du groupe contrôle, en particulier lorsqu'ils étaient scolarisés en enseignement prioritaire (Fischer et al., 2018). Nous disposons ainsi de certaines preuves statistiques de l'efficacité d'ACE ;

- l'ingénierie coopérative, puisqu'elle repose sur la co-construction de la progression par les professeurs et les chercheurs, fournit une grande partie de son travail à partir de films de la pratique. Ces films sont commentés et analysés, intégrés au sein de Ssystèmes Hybrides Texte-Image-Son (SHTIS ${ }^{27}$ ), et peuvent ainsi constituer des exemples emblématiques de la pratique ACE, dans lesquels des moments particuliers de la progression sont étudiés.

De tels exemples emblématiques, pensés, ainsi que nous l'avons mentionné ci-dessus, comme des exemples exemplaires (Kuhn, 1990) en devenir, peuvent constituer des preuves anthropologiques de l'efficacité de la pratique ACE. Ils peuvent aussi jouer un rôle dans la diffusion de la progression et des intentions et stratégies d'enseignement qu'elle requiert.

L'ingénierie coopérative ACE peut ainsi à la fois se concevoir comme un effort pour relier organiquement le travail conjoint de professeurs et de chercheurs dans la co-construction continue de pratiques d'enseignement plus efficaces (Joffredo-Le brun et al., 2018), et comme une tentative de conjuguer preuves statistiques et preuves anthropologiques dans l'appréhension de cette efficacité. Nous terminons cette contribution en reprenant rapidement ce dernier point.

\section{SYNERGIE ENTRE PREUVES STATISTIQUES ET PREUVES ANTHROPOLOGIQUES}

La production de preuves de type statistique représente selon nous un constituant majeur d'un système d'indices au sein desquels la production de preuves de type anthropologique (au sens que nous donnons à cette expression dans cette contribution) est tout aussi nécessaire. Ce qui corrobore selon nous un ensemble donné de conditions d'efficacité d'un enseignement repose sur l'identification d'indices à la fois statistiques et anthropologiques (Sensevy, 2014). C'est ce système qui va permettre de produire des assertions garanties (Dewey, 1938/1993, 1941) et de continuer à imaginer des pratiques d'enseignement qui les concrétisent ${ }^{28}$. 
Une fois un tel système d'indices initié, on peut conjecturer que la mise en œuvre d'une séquence d'enseignement présente une bonne probabilité d'obtention d'un ensemble d'effets - par exemple, pour ACE, une compréhension de l'arithmétique plus profonde, un rapport aux mathématiques plus fructueux pour le futur, une première appropriation de l'activité d'enquête mathématique, une première intégration de l'importance des représentations (publiques) dans la compréhension des mathématiques, etc. Une question cruciale est alors celle-ci : que va-t-il falloir qu'un professeur comprenne de la « pratique ACE », pour qu'il puisse reproduire l'effet voulu dans son contexte particulier? En particulier, que va-t-il devoir connaître de l'histoire de cette pratique, de sa genèse au sein du collectif de pensée qu'est $\mathrm{ACE}^{29}$ ? Pour le dire autrement, de quelle culture ( « visuelle» aussi bien que « narrative », « didactique » et « mathématique ») devrat-il devenir familier ? Quel arrière-plan devra-t-il progressivement construire et appréhender pour cela ? C'est dans cette perspective que les exemples emblématiques évoqués ci-dessus vont jouer un rôle crucial dans la pragmatique de la preuve. Il peut avoir été statistiquement démontré qu'une pratique donnée produit tel effet, mais l'effet attendu n'en sera pas obtenu, comme nous l'avons asserté ci-dessus, si la pratique n'est pas accomplie correctement, au-delà, pourrait-on dire, d'un certain seuil de justesse. La qualité, la puissance de la preuve, vont finalement dépendre de la qualité de la description de la pratique, qui devra elle aussi atteindre un certain seuil de justesse, et un certain seuil de concrétisation. Nous faisons l'hypothèse que c'est la manière dont on peut donner à voir et à comprendre une pratique, grâce à des instruments spécifiques ${ }^{30}$, qui rendra le professeur capable de comprendre cette pratique, tout en constituant la preuve, la garantie, de son efficacité.

On se souvient du mot fameux d'Engels (1880, publié en français en 1973, p. 35) : «La preuve du pudding, c'est qu'on le mange ${ }^{31} »$. Dans les sciences de la culture, manger le pudding, c'est un accomplissement, dont l'intégration demande une étude, souvent longue. La preuve de «l'air de la Reine de la Nuit », dans la Flûte enchantée, c'est qu'on l'accomplit, au terme - jamais vraiment atteint - d'un long processus d'intégration ${ }^{32}$.

Un point pour terminer. La présente contribution suppose en arrière-plan une certaine conception de la science, vue comme une ascension de l'abstrait au concret (Marx, 2008 ; Ilyenkov, 1982 ; Engeström et al., 2012). Dans les multiples implications que cette conception suppose, il en est une que nous voulons souligner. Le système de preuves, le système de garantie - dans lequel preuves anthropologiques et preuves statistiques s'établissent en synergie - que nous proposons de concevoir, est selon nous indissociable d'une pratique scientifique fondée sur la mise en réseau épistémique. Au-delà d'une seule recherche (par exemple d'une seule ingénierie coopérative), c'est la coopération organisée de collectifs divers et nombreux, hétérogènes dans leur composition, et progressivement de plus en plus collectivement capables, qui permettra, dans l'éducation comme c'est le cas en mathématique, en astronomie, ou en biologie (cf. Nielsen, 2011), de faire la science.

\section{NOTES}

1. Les auteurs de ce texte se sont appuyés sur le travail accompli dans un collectif de recherche attaché à développer la théorie de l'action conjointe en didactique, dont le livre Didactique pour enseigner (2019) exemplifie certains aspects, en relation étroite avec les arguments ici produits.

2. Il existe de très nombreuses études sur la question de l'evidence-based practice, dont beaucoup sont présentes dans l'article de Bryk et les contributions de ce dossier d'Éducation \& Didactique. On pourra également lire, en français, l'intéressante note de synthèse proposée par Frédéric Saussez et Claude Lessard dans la Revue française de pédagogie (Saussez et Lessard, 2009).

3. Barkham et Melior-Clark produisent ainsi « A cyclical model exemplifying the complementary relationship between evidence-based practice and practice-based evidence [...] presented as a means for furthering the delivery of a rigorous but relevant knowledge base for the psychological therapies. » (Barkham et Melior-Clark, 2003, p. 319 et 324). Sans forcément adhérer à la nature du modèle présenté, nous reviendrons sur cette question cruciale des relations entre les deux types de preuves à la fin de cette contribution. Green voit ainsi la practicebased research comme un " remède " à ce qu'il nomme "The "pipeline" fallacy of transferring research to practice » (Green, 2008, p. 20). Il affirme ainsi : "The pipeline conceptualization and imple- 
mentation of transferring research to practice results in successive constrictions of the flow of knowledge and an "evidence-based guideline" product at the practitioner end of the pipeline that has a poor fit with practice circumstances such as funding, time constraints and patient demands ». (C'est nous qui soulignons). Là non, plus, nous ne reprenons pas forcément à notre compte l'ensemble de cette assertion, en particulier, dans le champ de l'éducation, la nature des « circonstances » que Green décrit en substance. Toutefois, l'idée d'une " pauvre adéquation (poor fit) " aux circonstances de la pratique pour beaucoup d'evidence-based guidelines nous paraît essentielle.

4. Lidée qu'il faille accepter différents types de preuve, de même dignité épistémologique, est présentée avec force par David Hume, en défense de son Traité de la nature humaine : «It is common for Philosophers to distinguish the Kinds of Evidence into intuitive, demonstrative, sensible, and moral ; by which they intend only to mark a Difference betwixt them, not to denote a Superiority of one above another. Moral Certainty may reach as high a Degree of Assurance as Mathematical ; and our Senses are surely to be comprised amongst the clearest and most convincing of all Evidences. Now, it being the Author's Purpose, in the Pages cited in the Specimen, to examine the Grounds of that Proposition; he [David Hume, qui décrit lui même son ouvrage] used the Freedom of disputing the common Opinion, that it was founded on demonstrative or intuitive Certainty ; but asserts, that it is supported by moral Evidence, and is followed by a Conviction of the same Kind with these Truths, That all Men must die, and that the Sun will rise Tomorrow » (Hume, 1745, 2011, p. 426-427).

5. On pourrait probablement dire : sur la connaissance pratique de la logique de la pratique, au sens de Bourdieu (1980).

6. Cette dénomination peut prêter à discussion, bien entendu. Nous voulons mettre l'accent, ici, et comme on le verra dans ce commentaire, sur le fait que ce type de preuve provient de la connaissance pratique de la pratique. On pourrait appeler ce type de preuve preuve culturelle, tout aussi bien, pour souligner le fait qu'il suppose la connaissance d'une culture particulière, qui nourrit et " détermine» l'action étudiée.

7. On peut d'ailleurs noter que James utilise une nouvelle fois l'exemple du lever matinal dans le dernier chapitre de son livre, Talk to Teachers on Psychology, publié en 1899, soit 9 ans après ses Principles of Psychology. Là encore, l'exemple est pour lui un moyen de garantir sa théorie de la volition.

8. Ici, il faut entendre " garantir» au sens de l'assertabilité garantie de Dewey (1938-1993 ; 1941).

9. Les deux dates (1996 et 2014 renvoient à l'édition originale (1996) des Institutions du Sens, et à sa traduction de 2014 (The Institutions of Meaning. A Defense of Anthropological Holism). L'édition en anglais comporte une préface inédite de Vincent Descombes, au sein de laquelle il décrit en particulier le holisme anthropologique que son livre exprime et défend, holisme anthropologique qui nous semble au principe de ce que nous nommons preuve anthropologique, et que Descombes décrit ainsi, en référence à Fodor et LePore : "I have borrowed the term "anthropological holism" from Fodor and LePore, who use it to characterize Wittgenstein's position. They define it as follows: "Anthropological holism is distinct from semantic holism only insofar as it concerns the relation between language and its intentional background - that is, the relation between language and the cultural background of beliefs, institutions, practices, conventions, and so forth upon which, according to anthropological holists, language is ontologically dependent." Jerry Fodor and Ernest LePore, Holism : A Shopper's Guide (Oxford : Blackwell, 1992), page 6. »

10. On peut ainsi considérer les preuves statistiques, comme "des colosses (mathématiques) aux pieds d'argile ", les pieds d'argile, ici, étant représentés par la manière dont les unités qui forment la matière du calcul statistique sont ou non en adéquation avec la réalité que l'on cherche à comprendre et expliquer. D'un autre point de vue, on peut se rendre sensible aux risques de l'épistémologie de la «gouvernance par les nombres» (Supiot, 2015) qu'un certain usage de statistiques peut activer.

11. Nous entendons ici la notion de skill au sens de Ryle (1949), ou d'Ingold (2018).

12. Notons ici qu'on peut ne pas partager la réticence de P. Brook quant au langage. On peut penser au contraire qu'une description de telles pratiques est toujours possible, et même fructueuse - ce qui ne revient nullement à dire que le langage épuise la pratique.

13. Au sens où Bergson parle des « données immédiates de la conscience » (Bergson, 1888/2001), 
ce qui va l'amener à produire le concept de durée. Il nous semble important de saisir que les preuves anthropologiques reposent sur une conception du temps qui n'est pas celle du temps spatialisé, mais celle, précisément, de la durée de l'expérience. Il y a preuve anthropologique parce qu'il y a prise en compte de l'expérience accomplie dans une culture déterminée, expérience incompréhensible sans l'intégration de la durée.

14. C'est le cas non seulement dans la pratique quotidienne du travail scientifique, mais encore dans les théorisations des scientifiques les plus prestigieux, comme l'étudie par exemple Mario Livio (2014) dans son livre Brilliant Blunders : From Darwin to Einstein - Colossal Mistakes by Great Scientists That Changed Our Understanding of Life and the Universe.

15. Wikipedia (repéré à http://fr.wikipedia.org/ wiki/Giovanni_Morelli) décrit ainsi l'apport de Morelli : «Se distinguent deux méthodes d'attribution d'œuvres d'art à un peintre, la méthode synthétique qui se base sur la mise en rapport de l'ensemble des éléments d'un tableau (de Jonathan Richardson à Roberto Longhi) et la méthode analytique. C'est cette dernière que Morelli tâchera de mettre en valeur. Il s'agit de la technique du détail qui vise à distinguer la "manière" d'un artiste. Il estime que l'âme d'un peintre peut se copier mais pas les procédés matériels. Il s'agit d'une technique que l'on pourrait presque qualifier de scientifique. Il se méfie de l'impression générale et affirme qu'il faut au contraire isoler des détails pour effectuer une comparaison efficace de la morphologie humaine. Pour choisir ces détails, il faut sélectionner des objets qui offrent une certaine constance d'un tableau à l'autre. Il va alors s'attacher aux ongles et aux oreilles (détails qui ne sont pas appris en atelier comme peuvent l'être les yeux ou la bouche). »

16. Sur les trois princes de Serendip, cf. http:// fr.wikipedia.org/wiki/Voyages_et_aventures_des_ trois_princes_de_Serendip. Une preuve anthropologique semble ainsi le plus souvent ni vraiment inductive, ni vraiment déductive, mais plutôt abductive, dans le sens introduit par Peirce (repéré à http:// www.commens.org/dictionary/term/abduction). L'ensemble de ces " héros du paradigme indiciaire » exercent de fait une méthode abductive, qui amène à partir d'indices pour reconstruire une action, un comportement.

17. How the laws of physics lie, c'est le titre d'un livre publié en 1983 par Nancy Cartwright.

18. On peut trouver dans l'analyse de Tim Koschmann (2011, p. 5) des considérations qui nous paraissent essentielles quant à la considération de la pratique comme traitement : "Theories about instructional practice may be advanced and tested experimentally, but practice itself is never systematically studied in its own right. One consequence is that we are left functionally inarticulate with regard to the practical details of the very changes that we would like to see taken up by teachers. We need, therefore, to re-think our research priorities. Where the prevailing program focuses exclusively on improving outcome measures, we need to develop a new research agenda, one that addresses the importance of making competent instructional practice visible ». C'est nous qui soulignons. Nous reviendrons sur ce point.

19. C'est nous qui soulignons. La délibération, et plus encore le pouvoir de discernement (celui du connaisseur pratique) nous semble consubstantielle à la notion de skill, de capacité humaine.

20. Au sens scientifique du terme "modèle ", répétons-le.

21. Cf. un ensemble de travaux accomplis dans la théorisation et la mise en œuvre d'ingénieries coopératives : Sensevy (2011), Sensevy et al. (2013), Morales et al. (2017), Joffredo-Le Brun et al. (2017), Joffredo-Le Brun (2016), Morellato (2017), Jodry (2018), Lefeuvre (2018), Perraud (2018), Blocher (2018).

22. Le processus décrit ici peut s'inclure dans la catégorie générale de design-based research (Cobb et al., 2003), et présente certaines affinités avec la pratique des lesson studies (Elliot, 2015), celle de l'action research (Kemmis, 2009 ; Morales et al., 2017), ou celle du participatory design basedresearch (Penuel et al., 2011). Pour une première cartographie, cf. notamment Sensevy et al. (2013).

23. Réseau épistémique qui nous semble constituer une "communauté d'amélioration en réseau » (en anglais NIC : Networked Improvement Community) dont le principe a été proposé et décrit par Bryk (2017).

24. Division du travail qui pourrait renvoyer au dualisme antique, critiqué par John Dewey (par exemple 1920), entre la contemplation, la théorie (produite par les hommes libres dans la Grèce antique) et la pratique (la tâche des esclaves dans la Grèce antique). 
25. A contrario, on peut penser que l'une des raisons qui expliquent certaines difficultés des méthodes d'enseignement produites par la recherche à se concrétiser en séquences d'enseignement relativement « robustes » repose sur le maintien, au moins relatif, d'une division du travail entre « ceux qui pensent » et « ceux qui font». Cette division entraîne de facto, chez " ceux qui pensent ", l'attitude suivante : il suffit de dire à « ceux qui font » ce qu'ils doivent faire. Ceux qui font exécuteront.

26. Le site de la recherche ACE, repéré à http:// blog.espe-bretagne.fr/ace/ ; le site de l'ingénierie coopérative (LéA) associée à cette recherche, repéré à http://ife.ens-lyon.fr/lea/le-reseau/les-differents-lea/ reseau-ace-ecoles-bretagne-provence.

27. Pour un exemple exploratoire prototypique, « work in progress ", de tels systèmes hybrides texteimage-son (SHTIS), cf. http://pukao.espe-bretagne.fr/ public/tjnb/shtis_ace/reseau_analyse_approximation. html.

28. Pour ne prendre qu'un exemple, on peut utiliser des statistiques pour évaluer le degré d'accord d'un collectif de praticiens dans l'appréhension d'une pratique. Cela signifie alors qu'on mathématise ce degré d'accord, ce degré pouvant devenir un élément parmi d'autres - quoique précieux - pour comprendre la pratique et pour la transformer.

29. Knorr-Cetina (1999, p. 106) montre ainsi comment l'expérience se trouve encapsulée dans des histoires, au sein des collectifs scientifiques : «In addition to the experienced body, which silently remembers and performs, they are a kind of memory organ that laboratory practice depends on. There is, in the laboratories studied, another memory organ that preserves experience. This is the narrative culture of the laboratory-more specifically, stories. Stories are scenarios of former experiences that participants have had directly or have heard about. They are told and retold, on appropriate occasions, and thus travel through the laboratory. As long as they circulate, they keep the relevant experience alive, turning it into a sort of communal stock of knowledge... ». C'est nous qui soulignons. «Garder vivante l'expérience pertinente » est un élément crucial dans la perdurance et la transmission des pratiques postulées efficaces.

30. Notamment les systèmes hybrides texteimage-son (SHTIS) évoqués plus haut dont la première fonction consiste précisément à « donner à voir et à comprendre la pratique ». On retrouve ainsi la nécessité explicitée par Koschmann (2011, p. 5) : «making competent instructional practice visible».

31. Produite en anglais dans le texte français d'Engels: The proof of the pudding is in the eating.

32. En donnant à ce mot, ici comme dans l'ensemble de ce texte, d'ailleurs, le sens que lui donne Jean-François Billeter (2011), et qui renvoie à la production d'un geste juste.

\section{RÉFÉRENCES}

Billeter, J.-F. (2012). Un paradigme. Paris : Allia.

Barkham, M. et Mellor-Clark, J. (2003). Bridging evidencebased practice and practice-based evidence: developing a rigorous and relevant knowledge for the psychological therapies. Clinical Psychology \& Psychotherapy, 10(6), p. 319-327. Repéré à http://doi.org/10.1002/ cpp. 379 .

Bergson, H. (2007). Essai sur les données immédiates de la conscience ( $9^{e}$ éd.). Paris : PUF.

Blocher, J.-N. (2018). Comprendre et montrer la transmission du savoir: les systèmes hybrides textes-images-sons comme lieux de production et d'écriture de phénomènes. Illustrations en Théorie de l'action conjointe en didactique. Thèse de sciences de l'éducation. Brest : université de Bretagne occidentale.

Bourdieu, P. (1980). Le sens pratique. Paris : Éditions de Minuit.

Collectif didactique pour enseigner (CDPE) (2019). Didactique pour enseigner. Rennes : Presses universitaires de Rennes, à paraître.

Brousseau, G. (1998). Théorie des situations didactiques. Grenoble: La Pensée sauvage.

Bryk, A. S. (2017). Accélérer la manière dont nous apprenons à améliorer. Éducation et didactique, 11(2), p. 11-29.

Cartwright, N. (1983). How the laws of physics lie. Oxford : Clarendon.

Cartwright, N. (1989). Capacities and abstraction. Dans Philip Kitcher et Wesley Salmon (dir.), Scientific Explanation. Minneapolis : Univ of Minnesota, p. 349-356.

Cartwright, N. (1994). Nature's capacities and their measurement. Oxford : Clarendon Press.

Cartwright, N. (1999). The dappled world: a study of the boundaries of science. Cambridge: Cambridge University Press.

Cartwright, N. (2010). What Are Randomised Controlled Trials Good For? Philosophical Studies: An International Journal for Philosophy in the Analytic Tradition, 147(1), p. 59-70.

Certeau, M. de (2010). Arts de faire. Paris : Gallimard.

Cobb, P., Confrey, J., diSessa, A., Lehrer, R. et Schauble, L. (2003). Design Experiments in Educational Research. Educational Researcher, 32(1), p. 9-13. Repéré à http:// doi.org/10.3102/0013189X032001009. 
Descombes, V. (1996). Les institutions du sens. Paris : Minuit.

Descombes, V. (2014). The institutions of meaning: a defense of anthropological holism. Cambridge : Harvard University Press.

Dewey, J. (2014). Reconstruction en philosophie. Paris : Gallimard

Dewey (1993). Logique. Théorie de l'enquête. Paris : PUF.

Dewey, J. (1941). Propositions, Warranted Assertibility, and Truth. The Journal of Philosophy, 38(7), p. 169. Repéré à http://doi.org/10.2307/2017978.

Elliott, J. (2015). Towards a comprehensive pedagogical theory to inform lesson study: an editorial review. International Journal for Lesson and Learning Studies, 4(4), p. 318-327.

Engels, F. (1973). Socialisme utopique et socialisme scientifique. Paris: Éditions sociales.

Fischer, J.-P., Sander, E., Sensevy, G., Vilette, B. et Richard, J.-F. (2018). Can young students understand the mathematical concept of equality? A whole-year arithmetic teaching experiment in second grade. European Journal of Psychology of Education. Repéré à http://doi. org/10.1007/s10212-018-0384-y.

Fleck, L. (2005). Genèse et développement d'un fait scientifique. Paris: Les Belles Lettres.

Foucault, M. (1963). Naissance de la clinique. Paris : PUF.

Green, L. W. (2008). Making research relevant: if it is an evidence-based practice, where's the practicebased evidence? Family Practice, 25(Supplement 1), p. i20-i24. Repéré à http://doi.org/10.1093/fampra/ cmn055.

Ginzburg, C. (1987). Mythes, emblèmes, traces. Paris : Flammarion.

Hume, D. (2011). A treatise of human nature. Oxford : Clarendon Press.

Ilyenkov, E. (1982). The Dialectics of the Abstract and the Concrete in Marx's Capital. Moscow : Progress Publishers.

Ingold, T. (2018). L'anthropologie comme éducation. Rennes : Presses universitaires de Rennes.

James, W. (1892-1909). Précis de psychologie. Repéré à http://classiques.uqac.ca/classiques/james william/ precis de psychologie/precis de psychologie.pdf.

James, W. (2008). Talks to teachers on psychology and to students on some of life's ideals. Rockville, Md. : ARC Manor.

Jodry, G. (2018). Les affects dans la relation didactique. Une étude exploratoire en classe de sixième. Thèse de sciences de l'éducation. Brest : université de Bretagne occidentale.

Joffredo-Le Brun, S. (2016). Continuité de l'expérience des élèves et systèmes de représentation en mathématiques au cours préparatoire: une étude de cas au sein d'une ingénierie coopérative. Thèse de sciences de l'éducation. Brest : université de Bretagne occidentale.

Joffredo-Le Brun, S., Morellato, M., Sensevy, G. et Quilio, S. (2018). Cooperative engineering as a joint action. European Educational Research Journal, 17(1), p. 187208.

Kemmis, S. (2009) Action research as a practice based practice. Educational Action Research, 17 (3), p. 463-474.

Knorr-Cetina, K. (1999). Epistemic cultures: how the sciences make knowledge. Cambridge, Mass. : Harvard University Press.

Koschmann, T. D. (2011). Theorizing practice. Dans T. D. Koschmann (dir.) (2011). Theories of Learning and Studies of Instructional Practice. New York : SpringerVerlag New York Inc.

Kuhn, T. (1990). La tension essentielle. Paris: Gallimard.

Lefeuvre, L. (2018). Didactique de l'enquête pour une lecture interprétative d'une fable de Jean de La Fontaine, selon une épistémologie de l'abstrait au concret. Étude de cas au sein d'une ingénierie coopérative. Thèse de sciences de l'éducation. Brest : université de Bretagne occidentale.

Livio, M. (2014). Brilliant blunders: from Darwin to Einstein - colossal mistakes by great scientists that changed our understanding of life and the universe. New York: Simon \& Schuster.

Marx, K. (2008). Introduction à la critique de l'économie politique. Paris : LAltiplano.

Morales, G., Sensevy, G. et Forest, D. (2017). About cooperative engineering: theory and emblematic examples. Educational Action Research, 25(1), p. 128-139.

Morellato, M. (2017). Travail coopératif entre professeurs et chercheurs dans le cadre d'une ingénierie didactique sur la construction des nombres: conditions de la constitution de l'expérience collective. Thèse de sciences de l'éducation. Brest : université de Bretagne occidentale.

Munro, E., Cartwright, N., Hardie, J. et Montushi, E. (2017). Improving child safety: deliberation, judgment and empirical research. Durham : Centre for Humanities Engaging Science and Society.

Nielsen, M. A. (2014). Reinventing discovery: the new era of networked science. Princeton : Princeton University Press.

Penuel, W. R., Fishman, B. J., Cheng, B. H. et Sabelli, N. (2011). Organizing Research and Development at the Intersection of Learning, Implementation, and Design. Educational Researcher, 40(7), p. 331-337.

Perraud, C. (2018). Une ingénierie cooperative: des travailleurs, des professionnels et un chercheur dans le secteur du travail protégé (ESAT). Une enquête collective pour une amélioration des pratiques. Thèse de sciences de l'éducation. Brest : université de Bretagne occidentale.

Rizzolatti, G. et Sinigaglia, C. (2008). Les neurones miroirs. Paris : Odile Jacob.

Ryle, G. (1949). The concept of mind. London : Hutchinson's University Library.

Saussez, F. et Lessard, C. (2009). Entre orthodoxie et pluralisme, les enjeux de l'éducation basée sur la preuve. Revue française de pédagogie, 168, p. 111-136. Repéré à http://doi.org/10.4000/rfp.1804.

Sensevy, G. (1999). Éléments pour une anthropologie de l'action didactique. Note pour l'habilitation à diriger des recherches. Marseille : université de Provence. 
Sensevy, G. (2011). Le sens du savoir. Bruxelles : De Boeck. Sensevy, G. (2014). Characterizing teaching effectiveness in the Joint Action Theory in Didactics: an exploratory study in primary school. Journal of Curriculum Studies, 46(5), p. 577-610.

Sensevy, G., Forest, D., Quilio, S. et Morales, G. (2013). Cooperative engineering as a specific designbased research. ZDM, The International Journal on Mathematics Education, 45(7), p. 1031-1043.

Sensevy, G. et Santini, J. (2006). Modélisation: une approche épistémologique. Aster, 43, p. 163-188.

Sensevy, G., Tiberghien, A., Santini, J., Laubé, S. et Griggs, P. (2008). An epistemological approach to modeling: Cases studies and implications for science teaching. Science Education, 92(3), p. 424-446.

Supiot, A. (2015). La gouvernance par les nombres : cours au Collège de France, 2012-2014. Paris : Fayard. 